\title{
A Novel Sensor System for Mobile Robot Using Moire Technique
}

\author{
Hyunki Lee* and Hyungsuck Cho \\ Department of Mechanical Engineering, Korea Advanced Institute of Science and Technology, \\ Daejeon, South Korea
}

\begin{abstract}
Nowadays a major research issue of mobile robots is to develop a robust 3D environment sensing for navigation and task execution. To achieve this, a variety of techniques have been developed for the determination of the 3D scene geometric information such as stereo vision, laser structured light, laser range finder and so on. But these methods have many limitations. To overcome these limitations we introduce a new sensing algorithm, which is based on the moiré technique and stereo vision. To verify the performance of this sensor system we conducted a series of simulation for various simple environments. The result shows the feasibility of successful perception with several environments.
\end{abstract}

Keywords : 3-dimensional sensing, moiré technique, stereo vision, robot sensor system

\section{INTRODUCTION}

A major research issue for mobile robots is to develop an environment sensing and recognition system for mobile robot navigation and task execution, which is robust and efficient. A variety of techniques have been developed in the past for the determination of 3D scene geometric information. Especially, although the binocular vision sensors among them have been widely used as representative ones of passive visual sensors for mobile robots, they still suffer from image intensity variation due to the illumination noise, insufficient feature information on environment composed of plain surfaces, and correspondence problem between multiple images. These reasons have led most mobile robot researches on $3 \mathrm{D}$ environment reconstruction using visual sensors to deal with just straight line edge and corner as interesting features [1]-[3], but these features are saliently observed in well arranged and structured environment with polygonal objects or polygon-textured surfaces. In addition, this information is not sufficient to describe the whole structure of 3D space. Therefore, robots frequently use active sensors for more reliable range sensing, and have become recognized as a promising alternative proposal, which includes the infrared sensor, the ultrasonic sensor, and the laser sensor [4] In our previous research we developed the novel sensing algorithm based on laser structured light sensing method. [9].

In many approaches to indoor robot applications, laser sensor has been used for detail sensing and modeling objects, which is commonly categorized to the laser sensor and the laser range finder measuring the time-of-flight. Though the laser range finder has more advantages in views of measuring, it need still high cost with high power consumption, heavy weight, and low accuracy in near range. In addition, the latter needs more scanning procedure than the former. This scanning procedure is a time consuming task to limit the sensing time, and needs a precisely controlled scanning mechanism. In order to keep up the advantages of the sensor system using the laser-structured light and to decrease the sensing time without degradation of the sensor resolution, it was necessary to develop a new visual sensor system different from the sensors mentioned above.

To achieve this purpose we introduce new sensing algorithm based on moiré technique. Moiré technique is introduced in 1973 [5], which is based on the optical interferometric method. This method is well known that it can be measured 3D object shape quickly and accurately. It used the illumination such as laser, white light and so on, which is based on active vision sensor system, so the measurement result is robust to environment illumination noise and consequently accurate result is acquired. In addition to this sensing characteristic, the sensing time is very short, because it is based on the optical sensing method. As a result in these days it is used to many applications for measuring the 3D shape. With these advantages we think that the moiré technique can be adapted to mobile robot sensing system. But there is a limitation. The general moiré technique cannot acquire the absolute 3D information, because of 2- $\pi$ ambiguity which will discuss in section 2. To overcome this limitation we adapted the binocular camera system to the original moire technique. As we know, binocular camera system can acquire the absolute depth information. By combining binocular

* $\{$ hklee and hscho\}@1ca.kaist.ac.kr; phone +82-042-869-3253; fax +82-042-869-3210; 
camera system to original moiré technique we can obtain the absolute depth value for robot navigation and take execution.

\section{MOIRE TECHNIQUE}

Moiré technique is introduced at 1973 by H. Takasaki [5]. The representative methods of the moiré method are shadow moiré method and projection moiré method. The shadow moiré method can be used for small object. On the contrary to the shadow moiré method, the projection moiré method can be used for big objects. So we choose the projection moiré method for our task. In original projection moiré technique two gratings are fixed for given measurement system, so it is very difficult to change the fringe pattern flexibly. To overcome this limitation phase measuring profilometry (PMP) system is used [11][12]. In this section, we will discuss the principle of projection moiré technique, the PMP method which is an advanced method in moiré technique and the limitation of these methods.

\subsection{Moiré technique}

The moiré technique is to find the depth value of object surface by analyzing the moiré fringe pattern. [6] As shown in Figure 1(a), the hardware system of general moiré technique consists of light source, detector and two gratings which have same pitch. Among two gratings one located in front of the light source and one in front of the camera respectively. As shown in Figure 1(a), we can choose one arbitrary line $\mathrm{y}_{\mathrm{r}}$. We investigate this section at the top side and then the fringe pattern is observed as shown in figure 1(b). Here, we assume that the light wave projected to the object is a plane wave and detector is observed a plane wave.

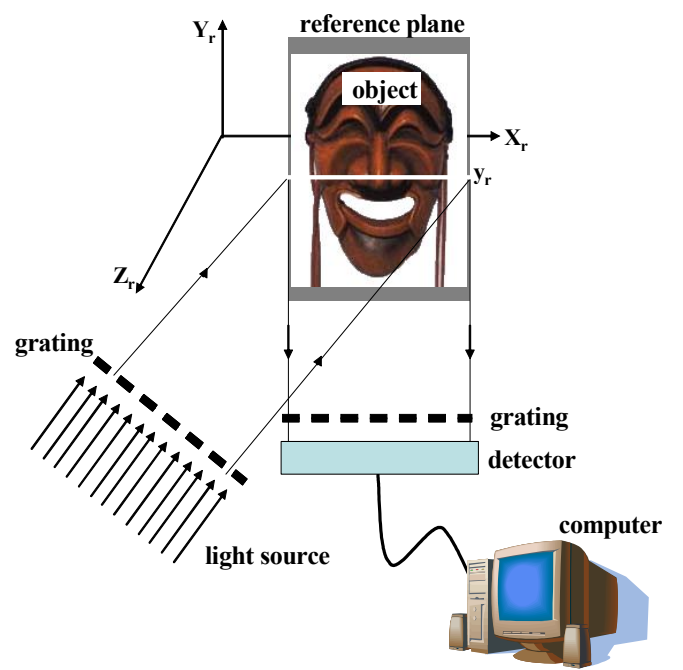

(a) hardware setup
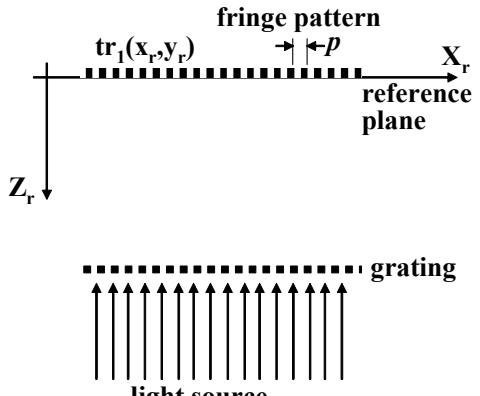

light source
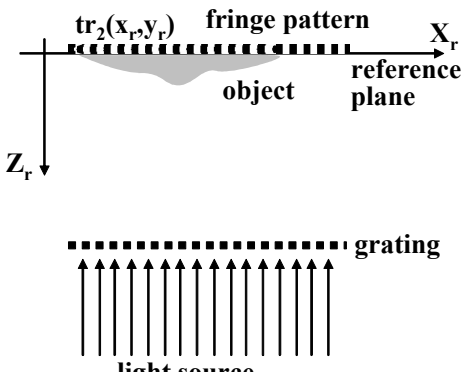

light source

(b) fringe pattern change

Figure 1. Hardware setup of the moiré technique

Gratings applied in moiré methods are transparencies with transmittances given by a square-wave function. Instead of square-wave functions, we describe linear gratings by sinusoidal transmittances bearing in mind that all types of periodic gratings can be described as a sum of sinusoidal gratings. As shown in the left image of Figure 1(b), if there is no object, the transmittance equation of constant frequency is given by

$$
t_{1}\left(x_{r}, y_{r}\right)=c+c \cos \left(\frac{2 \pi}{p} x_{r}\right)
$$

where $p$ is the pitch of a fringe pattern and the value of $c$ must be inside $0<\mathrm{c}<1 / 2$, which can be changed by power of light source. And $x_{r}$ and $y_{r}$ is the coordinate of the reference plane. The moiré pattern is projected onto the object. As 
shown in the right image of Figure 1(b), here the phase of the sinusoidal pattern $\operatorname{tr}_{l}\left(x_{r}, y_{r}\right)$ is modified by the optical path difference caused by the object as follows :

$$
t r_{2}\left(x_{r}, y_{r}\right)=c+c \cos 2 \pi\left(\frac{x}{p}+\psi\left(x_{r}\right)\right)
$$

$\psi\left(x_{r}\right)$ is the modulating the phase function and is equal to

$$
\psi\left(x_{r}\right)=\frac{u\left(x_{r}\right)}{p}
$$

where $u\left(x_{r}\right)$ is the displacement of the fringe line from the corresponding position of the fringe line formed at the reference plane as shown in Figure 2.

When the reference grating $t r_{l}$ locates onto the model grating $t r_{2}$, the resulting intensity becomes the sum of $t r_{1}+t r_{2}$. By addition we get

$$
\operatorname{tr}\left(x_{r}, y_{r}\right)=t r_{1}+t r_{2}=2 c\left\{1+\cos \pi \psi\left(x_{r}\right) \cos 2 \pi\left[\frac{x}{p}+\frac{1}{2} \psi\left(x_{r}\right)\right]\right\} .
$$

This information is obtained from the detector. By using this transmittance information, we do fast fourier transform for each row line. By doing frequency analysis we can extract the $\psi\left(x_{r}\right)$ information. [6] And then by using $\psi\left(x_{r}\right)$ information, we can estimate the distance $u\left(x_{r}\right)$ value in equation (3) and by using the geometry relating camera and projector, we can estimate the depth value of $z$.

$$
u\left(x_{r}\right)=z\left(\tan \theta_{1}+\tan \theta_{2}\right)
$$

where $\theta_{1}$ is an angle between light projection direction and $\mathrm{Z}_{\mathrm{r}}$-direction and $\theta_{2}$ is an angle between $\mathrm{Z}_{\mathrm{r}}$-direction and direction of camera's optical axis as shown in Figure 2.

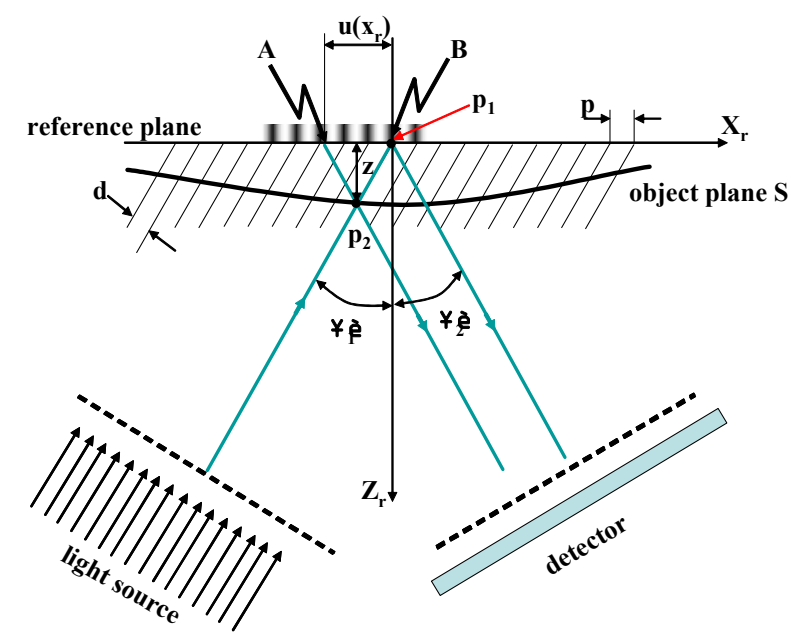

Figure 2. The fringe projection geometry of projection moiré 


\subsection{Projection moiré technique}

Figure 2 shows the principle of the projection moire technique. Here, to make a problem simple, we assume that the light wave projected to the object is a plane wave with oblique angle $\theta_{1}$.

The target of this method is to find the depth value $z$ of point $p_{2}$. If we can measure the distance value $u\left(x_{r}\right)$, the depth of $p_{2}$ can be estimated by using the geometry of the system. The distance value $u$ can be estimated from $\psi\left(x_{r}\right)$ in equation (3). In general moiré technique $\psi\left(x_{r}\right)$ value is obtained by coherence method as shown in equation (4). But in the projection moiré method $\psi\left(x_{r}\right)$ value can be obtained by geometric analysis as shown in Figure 2. In fact, the $\psi\left(x_{r}\right)$ values founded by general moiré technique and projection moiré technique are the same. [6]

Now we discuss about geometric method to find the phase modulation value $\psi\left(x_{r}\right)$. If there is no object, the intensity value of point $A$ is detected in the camera at a given pixel. If there is object plane(S), in the camera the intensity value of point $\mathrm{B}$ is detected at the given pixel. The intensity value difference between point $\mathrm{A}$ and $\mathrm{B}$ indicates the distance, $u\left(x_{r}\right)$, value. In equation (5), $\theta_{1}$ and $\theta_{2}$ is already given based on hardware architecture, and if $u\left(x_{r}\right)$ is given, we can estimate the depth value of point $p_{2}$ by using equation (5). [6] However, actually to make a plane wave for wide environment from illumination source is very difficult and generally detector has been used camera. Therefore, we consider another projection method with camera and lens as shown in Figure 3. [8]

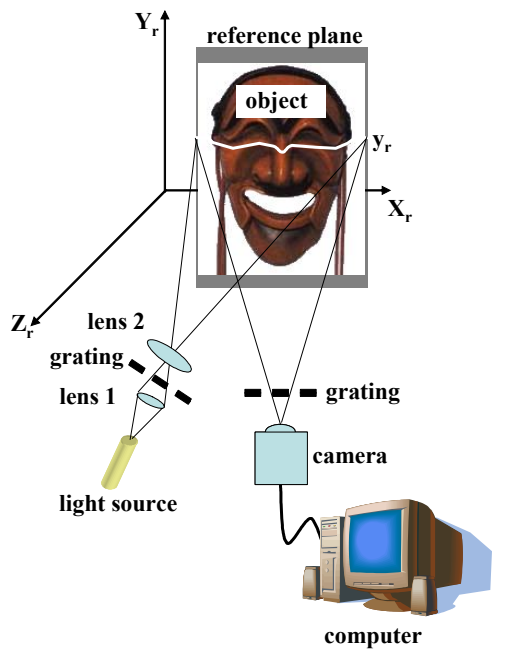

(a) fringe projection by means of grating imaging

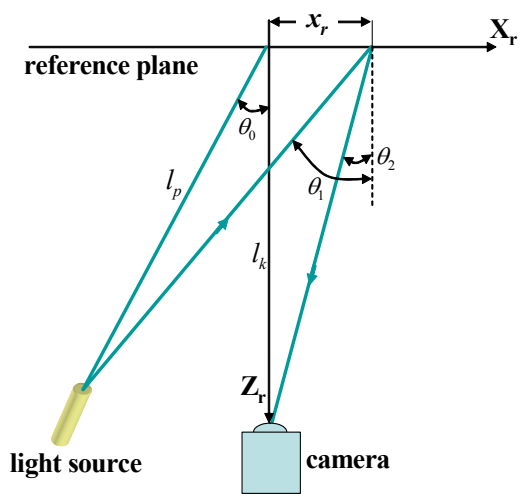

(b) fringe projection geometry

Figure 3. Hardware setup and geometry of projection moiré

In Figure 3(a), by using lens system the projection pattern which looks like a cone shape is projected to the object and the camera also has a perspective projection characteristic. If we choose one arbitrary line, $\mathrm{y}_{\mathrm{r}}$, in reference frame, along the white line in Figure 3(a) the $\mathrm{Y}_{\mathrm{r}}$-axis direction fringe pattern is defined and also we can know that along $\mathrm{Y}_{\mathrm{r}}$-axis the fringe pattern is changed. Here to adapt the moiré technique, we select one surface with the camera origin and $\mathrm{y}_{\mathrm{r}}$ line in the reference plane, which includes white line. Then we can obtain the geometric relation ship between a camera and light source as shown in Figure 3(b). In Figure 3(b), the dependence of $u$ and $\psi$ on the $\mathrm{X}_{\mathrm{r}}$-coordinate becomes

$$
\begin{aligned}
& u\left(x_{r}\right)=z\left(\tan \theta_{1}+\tan \theta_{2}\right)=z\left[\tan \theta_{0}+\frac{\left(l_{k}-l_{p} \cos \theta_{0}\right) x_{r}}{l_{p} l_{k} \cos \theta_{0}}\right] \\
& \psi\left(x_{r}\right)=\frac{z}{d}\left(\tan \theta_{1}+\tan \theta_{2}\right) \cos \theta_{1}=\frac{z}{d}\left[\sin \theta_{0}+\frac{\left(l_{k}-l_{p} \cos \theta_{0}\right) x_{r}}{l_{p} l_{k}}\right] \frac{l_{p}}{\sqrt{l_{p}^{2}+2 l_{p} x_{r} \sin \theta_{0}+x_{r}^{2}}}
\end{aligned}
$$

where $\theta_{0}$ is the projection angle measured from the $Z_{\mathrm{r}}$-axis and $l_{p}$ and $l_{k}$ are the projection and camera distances respectively. From equation (6) wee see that the displacement $u\left(x_{r}\right)$ becomes dependent on $x_{r}$ only through $\mathrm{z}$ if the projection lens and the camera lens are placed at equal heights above the $\mathrm{x}_{\mathrm{r}} \mathrm{y}_{\mathrm{r}}$-plane. 
The projection moiré technique has a good measurement resolution, but it has complicated hardware system and as shown in Figure 3 the pitch of two gratings of projection moiré technique is fixed. If we want to measure different environments with different depth information, we must change the pitch of two gratings and change the hardware setup. According to this it is very difficult to sensing for various environments flexibly. To overcome this inconvenience generally PMP method is used.

PMP system consists of laser diode, polygon mirror, cylindrical lens and photo diode as shown in figure 4. In Figure 4(a) the laser diode emits the light, cylindrical lens makes it slit beam [10], and the rotating polygon mirror reflects the light to the environment. Originally light emits as line by line, which is similar to scanning process using one laser source, but the projection speed is so fast that in the camera it looks as one regular fringe pattern. At this time as turning on and off the laser diode periodically and changing the angular velocity of polygon mirror, we can get different patterns flexibly. The sensing principle is the same as projection moiré technique as shown in Figure 4(b)

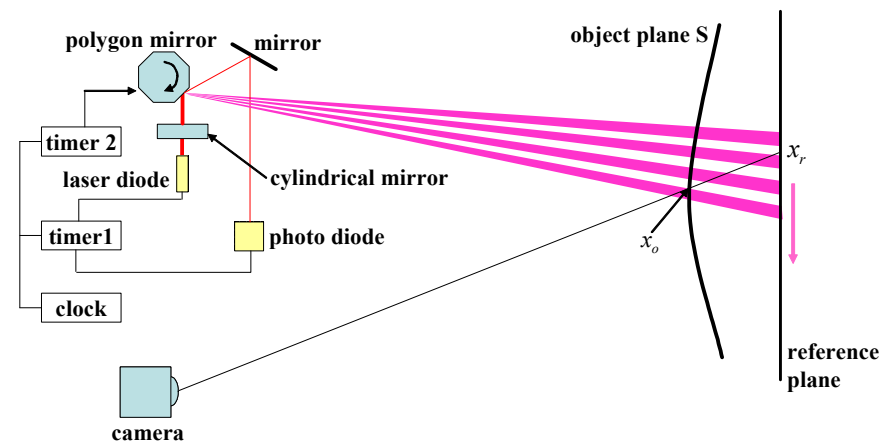

Figure 4. Hardware system and sensing principle of PMP

\subsection{Limitation of the projection moiré technique}

In section 2.1 we discussed about the measurement principle of projection moiré technique. By comparing the intensity value of reference plane with the intensity value caused by object surface, the displacement $u(x)$ value can be estimated and by using equation (5) we can estimate the depth value of the object surface. But this method has limitation at the phase difference estimation.

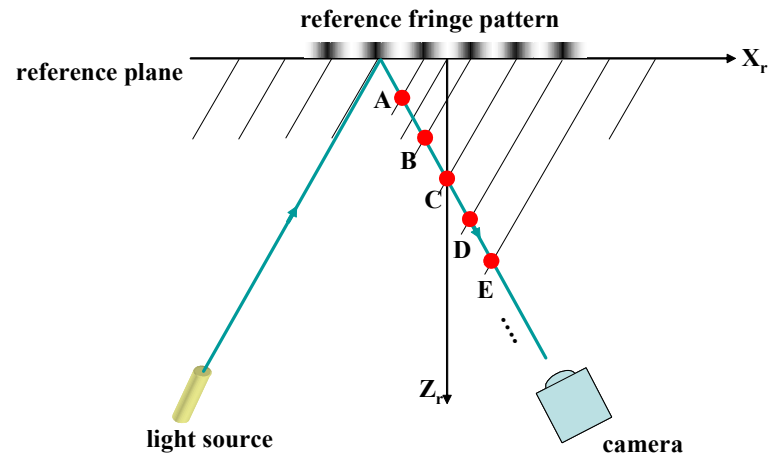

Figure 5. $2 \pi$-ambiguity of projection moiré technique

For example, as shown in Figure 5 the lowest intensity value is measured at given pixel in the camera. In this case A, B, C, D, E,... points in Figure 3 can become candidate points for depth measurement, which is based on projection moiré technique. Because projection fringe pattern appears periodically, this kind of phenomena occurs. It causes ambiguity for finding a depth. We called this $2-\pi$ ambiguity.

This description can be expressed by phase equation. If the phase difference between phase of reference plane and point $\mathrm{A}$ is $\varphi$, the phase of candidate points $\mathrm{B}, \mathrm{C}, \mathrm{D}, \mathrm{E}, \ldots$ can be written as follows : 


$$
\psi\left(x_{r}\right)=2 \pi N+\varphi \quad \text { for } \quad N=0, \pm 1, \pm 2, \pm 3, \ldots
$$

where $\psi\left(x_{r}\right)$ is the modulation function in equation (3), and $N$ is unknown, so we cannot decide corresponding point among them. Here $N$ expresses the $2-\pi$ ambiguity.

\section{PROPOSED ALGORITHM FOR 3D SENSING}

In section 2.3 we discussed about limitation of typical projection moiré technique. To overcome this limitation we suggest a new sensing architecture and algorithm based on moiré technique and stereo vision technique.

\subsection{Architecture of the proposed algorithm}

As shown in Figure 6(a), our proposed sensor system consists of two cameras and one multi-stripe laser projector. The sensing principle is based on moiré technique and stereo vision.

Originally the moiré technique has a 2- $\pi$ ambiguity. We can express it as equation (7). But in our proposed algorithm which used two cameras we can make two phase modulation equations as follows:

$$
\begin{array}{ll}
\psi_{L}\left(x_{r}\right)=2 \pi N_{L}+\varphi_{L} & \text { for } N_{L}=0, \pm 1, \pm 2, \pm 3, \ldots \\
\psi_{R}\left(x_{r}\right)=2 \pi N_{R}+\varphi_{R} & \text { for } N_{R}=0, \pm 1, \pm 2, \pm 3, \ldots
\end{array}
$$

where $\psi_{L}\left(x_{r}\right)$ and $\psi_{R}\left(x_{r}\right)$ are the modulation function at a given point, $\varphi_{L}$ is the smallest phase shifting value among candidate points at given point in the left camera and $\varphi_{R}$ is the smallest phase shifting value among candidate points at given point in the right camera. Here $\varphi_{L}$ and $\varphi_{R}$ can be measured by the moiré technique and the depth value $z$ in Figure 2 which is estimated by $\psi_{L}\left(x_{r}\right)$ and $\psi_{R}\left(x_{r}\right)$ must be same. Then, in equation (8) if we find the integer $N_{L}$ and $N_{R}$ respectively which satisfy the equation (9) with measured $\varphi_{L}$ and $\varphi_{R}$, we can solve the equation (8) and also can overcome the $2-\pi$ ambiguity problem.

\subsection{Stereo vision}

Figure 6(b) shows a simple stereo vision system and this stereo architecture is easier to handle the image data than the architecture of general stereo vision. Here two optical axes of the camera are parallel and two cameras' intrinsic parameter are the same. We assume that the $x_{l i}$ in the left intensity image and $x_{r i}$ in the right intensity image are correspondence points for point P. Here $i$ means the $i^{\text {th }}$ correspondence point at given scan line. The depth value of $\mathrm{P}$ can be estimated from

$$
z=\frac{T f}{x_{l i}-x_{r i}} .
$$

where $T$ is the distance between two camera and $f$ is focal length of the camera. If we find correspondence points in the left and right intensity information and the intrinsic and extrinsic camera parameters are given, we can estimate the depth value $\mathrm{z}$ of point $\mathrm{P}$ by using equation (9). We call the denominator of the equation (9) disparity, $d$.

$$
d=x_{l i}-x_{r i} .
$$

This disparity value must be over zero as shown in Figure 7(b). This is important characteristic of stereo vision. There is some constraint of the stereo vision technique, where the disparity characteristic is included.

$$
\begin{aligned}
& \text { Constraint 1: } 0 \leq x_{l i}-x_{r i} \\
& \text { Constraint 2: } x_{l i}<x_{l j} \text { and } x_{r i}<x_{r j}, \quad 1 \leq i<j
\end{aligned}
$$




$$
\text { Constraint 3: } x_{l i+1}=x_{l i}+1 \text { or } x_{r i+1}=x_{r i}+1
$$

The constraint 1 indicates the characteristic of the positive disparity while the constraint $\mathrm{C} 2$ means that each correspondence point must be in order at left and right intensity information respectively. The constraint 3 deals with the occlusion. If some area of left intensity image is occluded, equation $x_{r i+1}=x_{r i}+1$ is adapted and then we find the correspondence point which satisfies the constraint 2 . On the contrary, $x_{l i+1}=x_{l i}+1$ is adapted and also we find the correspondence point which satisfies the constraint 2.

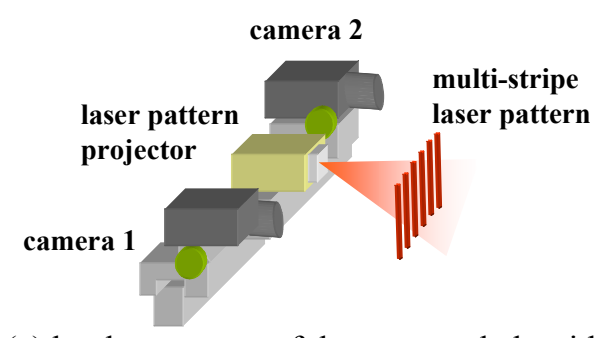

(a) hardware setup of the proposed algorithm

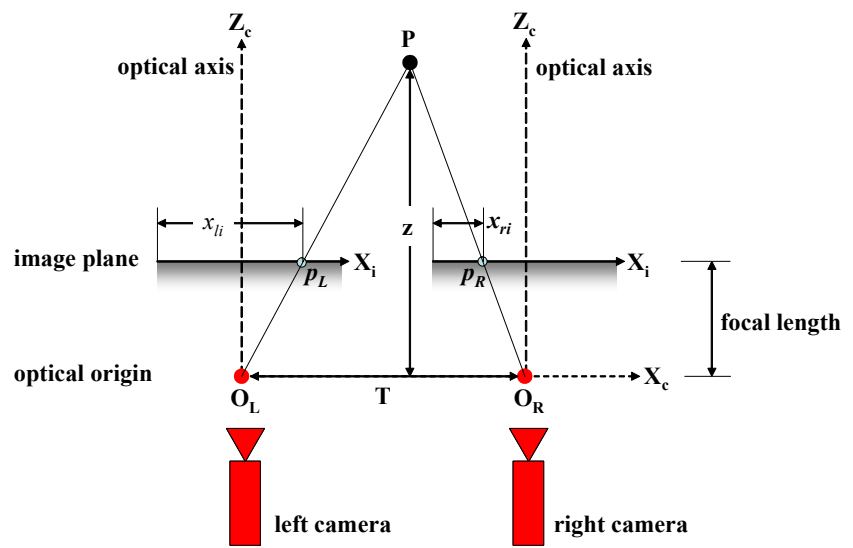

(b) special stereo vision architecture

Figure 6. The architecture of general stereo vision

Generally, to find the correspondence in the two stereo images is difficult and many researches have been conducted. The related researches can be roughly classified into local method and global method. [14] A representative one utilizes the area-based intensity correlation method between the right and left images. Among a variety of similarity measures between two windows specified in the left and right images, a simplest measure called SAD (sum of absolute differences), SSD (sum of squared differences), NCC (normalized cross correlation), or SMW (symmetric multiple window) has been widely used. [15] While they are efficient, they are sensitive to local intensity noise (occlusion or uniform textured region). In contrast, global methods can be less sensitive to these problems since global constraints such as depth continuity constraints provide additional support for these ambiguous regions. However, it results in expensive computational costs. As a global method, dynamic programming and graph cuts are frequently used. A detail description for current trend of stereo vision research can be found in the reference. [13][14]

\subsection{Sensing algorithm of the proposed algorithm}

The schematic hardware system which we have discussed in section 3.1 is shown in Figure 7(a). The world coordinate locates at the center of the left camera and the center point of pattern projector locates at $\mathrm{P}_{1}$. As shown in the figure, the reference fringe pattern is generated by pattern projector. If object locates in front of the reference plane, the slightly changed fringe pattern images in the left and right image can be acquired as shown in Figure 7(a). Here, we choose one same row line in the left and right image: a-a' in the left image and b-b' in the right image, which is the epipolar line for each other.

The first step of the proposed algorithm is to search the candidate points for depth measurement based on the moiré technique. Here the pattern projector and right camera are used. We choose one fringe edge pixel at given scan line b-b'. Then according to geometric relationship we can find the candidate points by using moiré technique. For example, as shown in Figure 7(a), we can select one point, $\mathrm{R}_{1}$, which is right side fringe pixel. Then we can find the line equation of $1_{R_{1}}$ by using camera equation. Here, $1_{R_{1}}$ is $O_{R} A$. In general $3 \mathrm{D}$ case to express the line equation is very difficult, so the plane which consists of right optical origin, scan line and given reference pattern can be obtained and we can make 2-D plane. In this case, the equation for $1_{R_{1}}$ is simply estimated by: 


$$
Z=\frac{M_{R}(1,1)}{x_{R_{1}}-M_{R}(1,3)} X+\frac{M_{R}(1,4)}{x_{R_{1}}-M_{R}(1,3)}
$$

where $x_{R_{1}}$ is the $\mathrm{X}$-axis coordinate of the pixel $\mathrm{R}_{1}$ in the right image plane and $M_{\mathrm{R}}(\mathrm{i}, \mathrm{j})$ is the element of $\mathrm{i}^{\text {th }}$ row and $\mathrm{j}^{\text {th }}$ column of the right camera projection matrix $M_{\mathrm{R}}$. In this way, we can derive the simple line equation $1_{R_{1}}$ independent of the Y-coordinate. Then we try to find the candidate points for depth measurement by using moiré technique: The candidate points are defined as the intersection points of two lines; one line of which is line $1_{R_{1}}$ and second line of which connects $P_{1}$ and fringes which share the same fringe edge as $R_{1}$ 's in the reference fringe pattern. The candidate points $(A, B, C, D, \ldots)$ are shown in Figure 7(a).

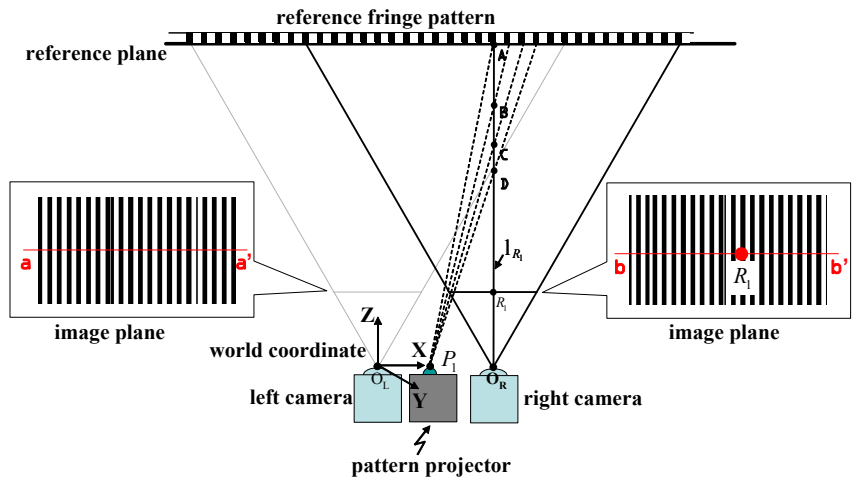

(a) First step of the proposed algorithm based on the moire technique

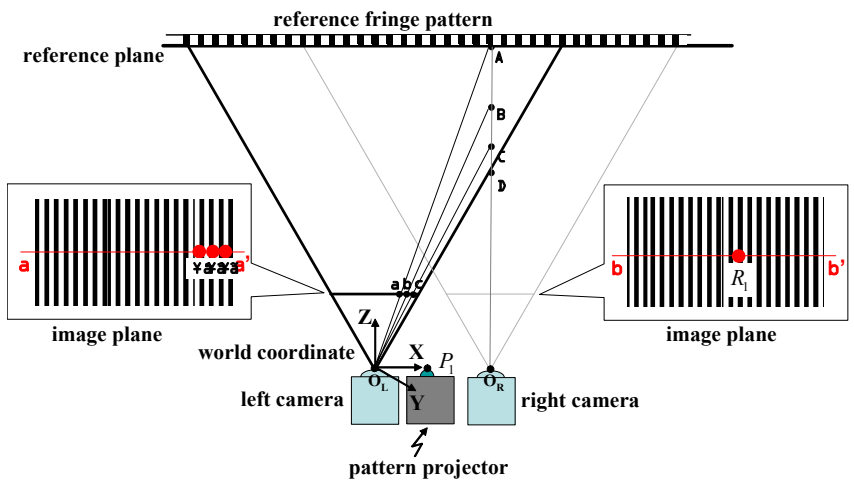

(b) Second step of the proposed algorithm based on the stereo vision technique

Figure 7. The proposed algorithm based on the moiré technique and stereo vision

The second step is based on stereo vision to find the depth value by searching the equivalent corresponding to a given point in the right image based on stereo vision principle. In the previous step we found the candidate points by using moiré technique. In this process we projected these points into the image plane of left camera. Figure 7(b) shows this process briefly. We projects the candidates points A, B, C, D, .. to the image plane of left camera. Then we can get the corresponding projected points $\alpha, \beta, \gamma$. In point $\mathrm{D}$ case, the projected point is out of the image plane of left camera, so the point $\mathrm{D}$ is discarded for consideration. The following left camera projection matrix is used for the process. The equation comes from camera projection matrix. [16]

$$
S\left[\begin{array}{c}
x_{L} \\
y_{L} \\
1
\end{array}\right]=M_{L}\left[\begin{array}{c}
X_{\text {cand }} \\
Y_{\text {cand }} \\
Z_{\text {cand }} \\
1
\end{array}\right]
$$

where $s$ is scale factor, $X_{\text {cand }}, Y_{\text {cand }}$ and $Z_{\text {cand }}$ are the coordinates of the candidate points, $(\mathrm{A}, \mathrm{B}, \mathrm{C}, \mathrm{D}, \ldots)$ in the world coordinates, $x_{L}$ and $y_{L}$ are the image coordinate in the left camera and $M_{L}$ is $3 \times 4$ camera projection matrix of left camera, which consists of intrinsic and extrinsic parameters.

After projecting all candidate points onto the left image, we compare the intensity value of the projected points in the left image with that of point $\mathrm{R}_{1}$ in the right image. According to the stereo vision principle, the correspondence point should have the same intensity value; if $R_{1}$ is located at the left side edge pixel of the fringe, so is the correspondence point; if $R_{1}$ is the right side edge pixel of the fringe, so is the correspondence point. In Figure 7(b) the point $\alpha$ has the same intensity value as that of the given point $R_{1}$ and is also located in the same side edge pixel of the fringe as $R_{1}$. On the contrary, the other two points $\beta$ and $\gamma$ have different intensity values from that of the given point $R_{1}$ and are not located in the same side edge pixel of the fringe as $R_{1}$. According to this result, we know that pixel $R_{1}$ in the right image 
and $\alpha$ in the left image are corresponding to each other. By using the $\mathrm{X}_{\mathrm{i}}$-coordinate of pixels $\mathrm{R}_{1}$ and $\alpha$ in the image coordinate, we can estimate the depth value of point A from equation (9).

\subsection{Dynamic programming}

In this paper to find the correspondence point between two left and right fringe pattern images, we adapted the dynamic programming method. [7] This dynamic programming method is originally adapted at stereo vision to find the optimal corresponding points' set between left and right image. In this paper this method is adapted to find the correspondence between left and right fringe patterns.

By scanning each left and right side fringe edge in given scan line, the optimization process is performed with the following cost function:

$$
J=N_{o c} \lambda_{o c}-N_{m} \lambda_{r}+\sum_{i=1}^{N_{m}} d\left(x_{i}, y_{i}\right)
$$

where $J$ is the cost function in given scan line, $\lambda_{o c}$ is the constant occlusion penalty, $\lambda_{r}$ is a constant match reward, and $N_{o c}$ and $N_{m}$ are the number of occlusions (not the number of occluded pixels). And $d\left(x_{i}, y_{i}\right)$ is the dissimilarity between pixels $x_{i}$ and $y_{i}$ at scan line. We define $d\left(x_{i}, y_{i}\right)$ as follows:

$$
d\left(x_{i}, y_{i}\right)=\left\{\begin{array}{cc}
0 & \text { if } x_{i} \text { and } y_{i} \text { are correspondence point } \\
\lambda_{\text {mat }} & \text { if } x_{i} \text { and } y_{i} \text { are up-fringe } \\
\lambda_{\text {mat }} & \text { if } x_{i} \text { and } y_{i} \text { are down-fringe } \\
\text { penalty } & \text { otherwise }
\end{array}\right.
$$

where $\lambda_{\text {mat }}$ is matching value when $x_{i}$ and $y_{i}$ is the same fringe style, which is less than penalty value and bigger than the constant occlusion penalty $\lambda_{o c}$, and penalty value is very big value to prevent to matching.

\section{SIMULATION RESULT}

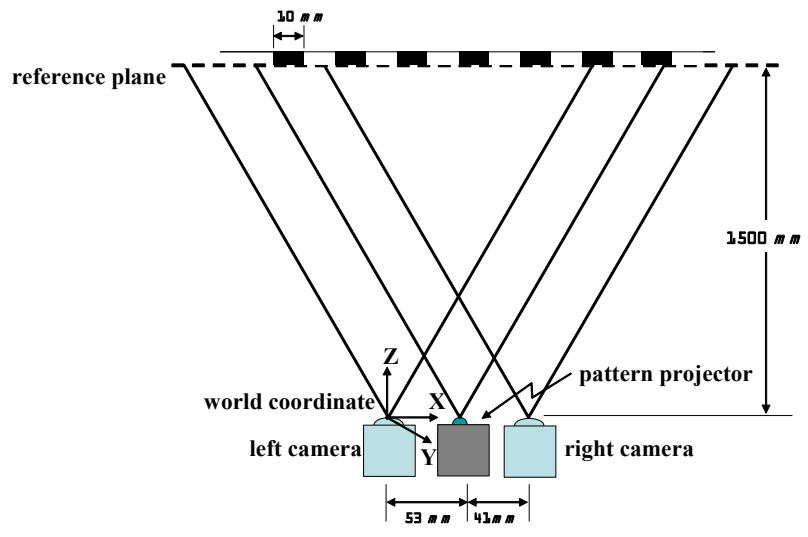

(a) hardware setup for simulation

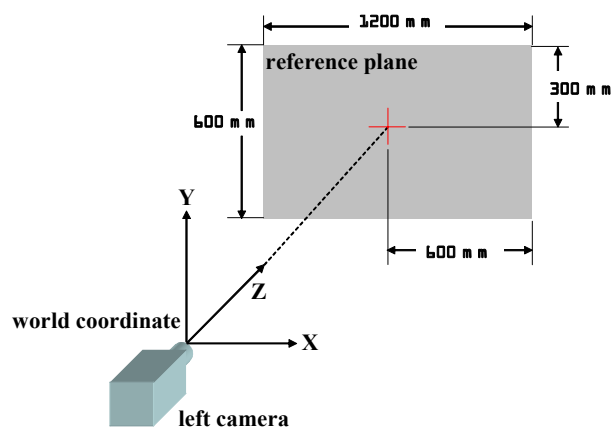

(b) relationship between camera and reference plane

Figure 8. Hardware and coordinate setup for simulation

For simulation we set up the hardware system as shown in Figure 8(a). The world coordinate locates at the center of left camera and we put the optical axis of the right camera parallel with the optical axis of the left camera. The distance between left camera and projector, and projector and right camera is $53 \mathrm{~mm}$ and $41 \mathrm{~mm}$ respectively. The reference plane locates at $1500 \mathrm{~mm}$ from the sensor system and the center of reference plane locates the Z-axis of world coordinate as shown in Figure 8(b). We set up that the left and right camera have same intrinsic parameter as follows; 


$$
M_{\mathrm{int}}=\left[\begin{array}{ccc}
\alpha & -\alpha \cot \theta & u_{0} \\
0 & \frac{\beta}{\sin \theta} & v_{0} \\
0 & 0 & 1
\end{array}\right]=\left[\begin{array}{ccc}
1249.89117 & 0 & 323.54041 \\
0 & 1254.91338 & 221.62866 \\
0 & 0 & 1
\end{array}\right]
$$

where $\alpha$ is $k f, \beta$ is $l f, k$ and $l$ are the scale parameter of the CCD unit respectively, $f$ is focal length of camera, $\theta$ is skew angle of CCD cell and $u_{0}$ and $v_{0}$ are the $\mathrm{X}_{\mathrm{i}}$ - and $\mathrm{Y}_{\mathrm{i}}$ - coordinates of the center point of the image plane. These parameters can be acquired by camera calibration. [16]

The fringe pattern which has $20 \mathrm{~mm}$ inter-fringe length is projected onto object.

First of all we investigate the fringe pattern change according to the object shape. First, we investigate the relationship between object distance and fringe pattern. We put a flat board in front of the reference plane. Then we change the distance of the flat board from $1000 \mathrm{~mm}$ to $600 \mathrm{~mm}$ at each $50 \mathrm{~mm}$. Then we observed the fringe pattern change at the right camera.

\begin{tabular}{|l} 
distance from a flat board to camera \\
$1000 \mathrm{~mm}$
\end{tabular}

Figure 9. The fringe changes according to the object distance change

As shown in Figure 9 the distance of the flat board becomes closer and closer to the sensor system, the fringe pattern goes to left and left and especially at the distance $700 \mathrm{~mm}$ the fringe pattern is similar to the $1000 \mathrm{~mm}$.

Next we investigate the fringe pattern change when occlusion occurs. We put a small flat object in front of the reference plane and change the distance between reference plane and a flat object. As shown in Figure 10(b) the fringe pattern inside dotted square are changed. Especially the part of patterns which belong to the object looks like going to the left side and the part of patterns which belong to the reference plane looks like disappearing the fringe pattern.

Finally we investigate the fringe pattern change, when object shape is convex curved. Here we compare the fringe pattern for curved object with it for reference plane as shown in Figure 10(d). At right side of the pattern frequency is longer than it of reference plane and at the left side of the pattern frequency becomes shorter.

Based on these tendencies of the fringe pattern changes according to the object shape we simulated to the various environments. With the hardware assumption which illustrated in Figure 8, we simulated various objects to verify the proposed algorithm.

First, as shown in figure 11, we simulated free form object. In the figure we can see the moiré pattern acquired from left and right camera and can see the simulation result. In figure 11(c) and (d) we can see the moiré pattern in the intensity image at left and right cameras. In these figures we can see that the frequency of pattern is increase and decrease depending upon the object shape. By using these two images and proposed algorithm, we can get a 3D depth result as shown in Figure 11(b). We can see the sine curve shape clearly. The 3-D result data is point's cloud, so in Figure 11(b) we can observe some spaces among 3-D depth result.

We also simulate the proposed algorithm to other various environments. In Figure 12(a) cylindrical and square object is located in the center of reference plane. We can see that the modified fringe patterns as shown in Figure 12(c) and (d) and in Figure 12(b) we can see the result of 3D measurement by using our proposed algorithm. In the 3-D depth map image, two objects, square object and cylindrical object, are shown clearly. Originally the moiré technique cannot find the accurate depth of occlusion object, because of $2 \pi$-ambiguity. On the contrary this, our proposed algorithm can find the accurate depth though occlusion object. In Figure 12(b) shows this result. We can see the shape of the square object and the accurate position of square object. 


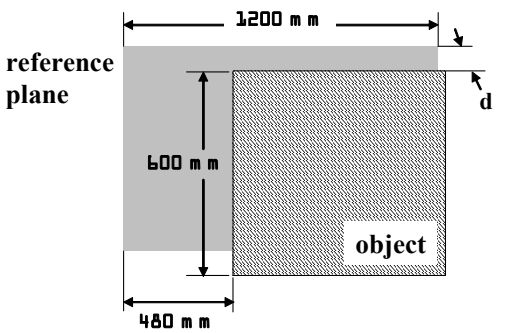

(a) occlusion object

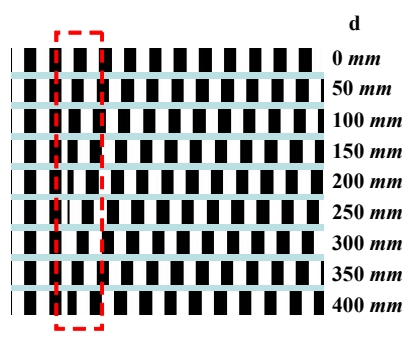

(b) fringe pattern change for occlusion object

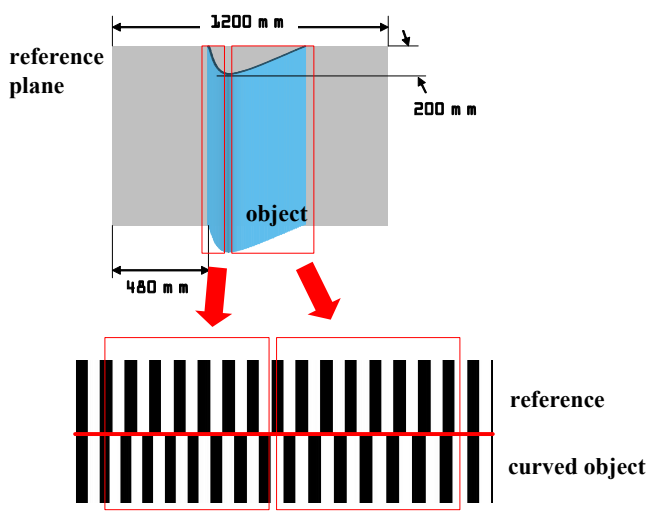

(c) curved object

(d) fringe pattern change for curved object

Figure 10. The fringe changes for curved object

\section{CONCLUSION}

A major research issue associated with mobile service robots is the creation of autonomous environment sensing and recognition system for navigation and task execution that is robust and efficient. In this work, we proposed a new sensing algorithm based on the moiré technique and stereo vision for this sensor system acquiring more reliable range information. Since original projection moiré technique has a limitation for 3 dimensional environments sensing, the proposed algorithm combined with the stereo vision technique.

To see how the proposed algorithms can be applied to applications, we simulated in various environments. The performed simulations show the feasibility of successful perception of the mobile robot with the proposed sensor algorithm on several environments.

This research is now going on progress. The algorithm needs more improvements for applying to a mobile robot system. Especially sensing speed is important problem for applying our algorithm to a mobile robot system. To improve the sensing speed, at first time we adapt our algorithm for some rows of the fringe image, and then for neighborhood lines we find the depth by comparing the fringe between neighborhoods row. In addition to this, by adapting the DSP system it can improve the sensing speed.

\section{ACKNOWLEDGEMENT}

This work was supported by the Ministry of Information \& Communications, Korea, under the Information Technology Research Center (ITRC) Support Program.

\section{REFERENCES}

1. O. Faugeras, Three dimensional computer vision: A geometric viewpoint, MIT Press, 1993.

2. N. Ayache, Artificial Vision for Mobile Robots: Stereo vision and Multisensory perception, MIT Press, 1991.

3. P. Weckesser and R. Dillmann, Modeling unknown environments with a mobile robot, Robotics and Autonomous Systems, vol. 23, pp. 293-300, 1998.

4. H. R. Everett, Sensors for Mobile Robots: Theory and Application, AK Peters, 1995. 
5. H. Takasaki, Moire Topography, Applied optics, v.12, no.4, pp.845-850, 1973.

6. K.J. Gåsvik, Optical Metrology, $2^{\text {nd }}$ edition, John Wiley \& Sons Ltd, 1995.

7. S. Birchfield and C. Tomasi, Depth discontinuities by Pixel-to-Pixel Stereo, International Journal of Computer Vision, Vol. 35, No. 3, pp. 269-293, 1999.

8. J.F. Cardenas-Garcia, S. Zhang and F.Z. Shen, Implementation and use of an automated projection Moire experimental set-up, Optics and Lasers in Engineering, v. 21, pp. 77-98, 1994.

9. M. Y. Kim, H. S. Cho and H. K. Lee, "An Active Trinocular Vision System for Sensing Mobile Robot Navigation Environments," IEEE/RSJ International Conference on Intelligent Robots and Systems, 2004.

10. Website : http://repairfaq.ece.drexel.edu/sam/CORD/leot/course06_mod08/mod06-08.html

11. K. Creath, Phase-Measurement Interferometry Technique, Progress in Optics, Vol. 26, pp. 349-398, 1988

12. M. Kusawinska, Use of phase-stepping automatic fringe analysis in moiré inteferometry, Applied Optics, 1987

13. D. Scharstein and R. Szeliski, "A Taxonomy and Evaluation of Dense Two-Frame Stereo Correspondence Algorithms," International Journal of Computer Vision, Vol. 47, pp. 7-42, 2002.

14. M. Z. Brown, D. Burschka, and G. D. Hager, "Advances in computational stereo," IEEE Trans. Pattern Analysis and Machine Intelligence, Vol. 25, No. 8, pp. 993-1008, 2003.

15. E. Trucco, A. Verri, Introductory Techniques for 3-D Computer Vision, Prentice Hall, 1998.

16. D. A. Forsyth and J. Ponce, Computer Vision: A Modern Approach, Prentice Hall, 2003.

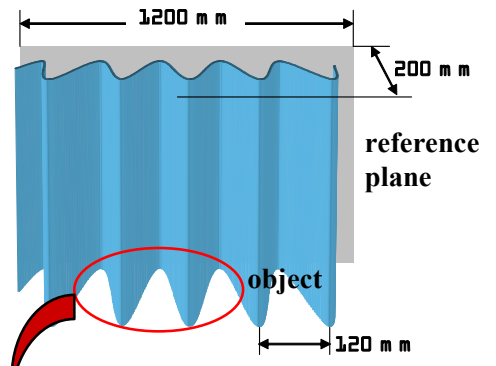

(a) free form object

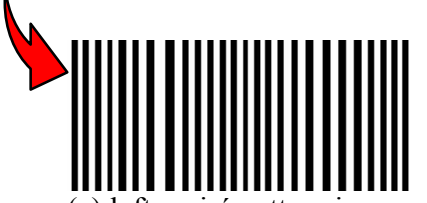

(c) left moiré pattern image

Figure 11. Experimental result for test environment 1
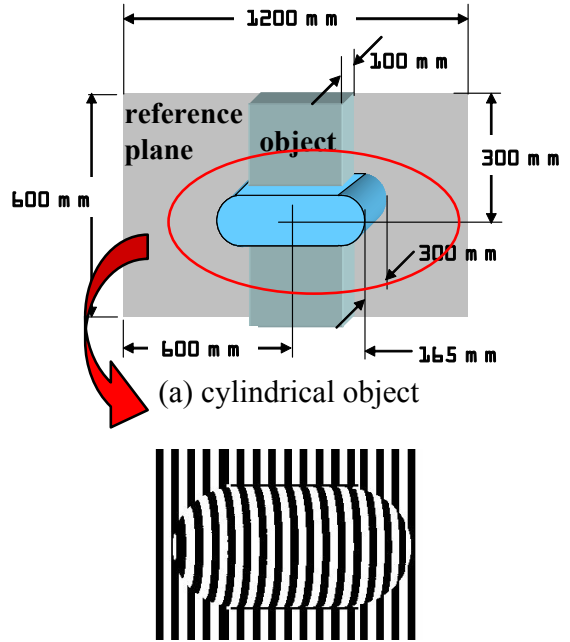

(c) left moiré pattern image

Figure 12. Experimental result for test environment2

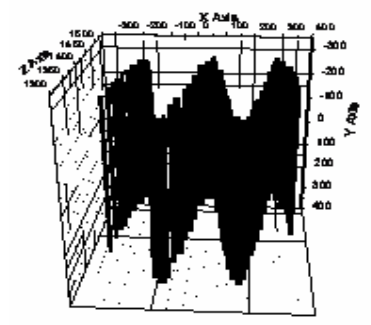

(b) measurement result

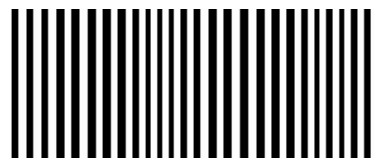

(d) right moiré pattern image

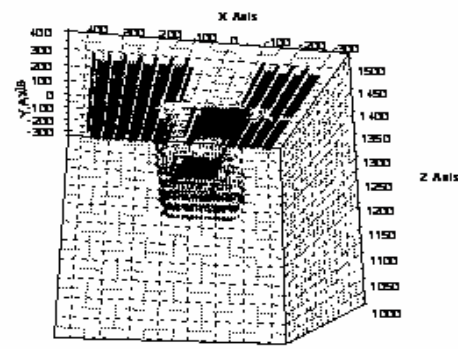

(b) measurement result

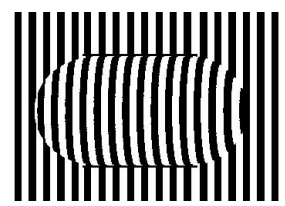

(d) right moiré pattern image 